\title{
CONCATENATIONS APPLIED TO ANALYTIC HYPOELLIPTICITY OF OPERATORS WITH DOUBLE CHARACTERISTICS
}

BY

\author{
KIL HYUN KWON
}

\begin{abstract}
We use the method of concatenations to get a sufficient condition for a class of analytic pseudodifferential operators with double characteristics to be analytic hypoelliptic.
\end{abstract}

Introduction. The present paper is concerned with analytic hypoellipticity for operators on an $N$-dimensional real-analytic manifold $\Omega$, of the form

$$
P(x, D)=I_{d} p(x, D)+Q(x, D),
$$

where $I_{d}$ is the identity $d \times d$ matrix, $p(x, \xi)$ a scalar analytic symbol, homogeneous of degree $m$ in $\xi$, and $Q(x, D)$ a $d \times d$ matrix of classical analytic pseudodifferential operators of order $m-1$ ("classical" means that its total formal symbol is a series of homogeneous terms whose homogeneous degrees drop by integers). We assume that its principal symbol, $p(x, \xi)$, is nonnegative everywhere, that it vanishes exactly of order two on its characteristic set $\Sigma$, and that $\Sigma$ is a symplectic real-analytic submanifold of $T^{*} \Omega \backslash 0$. For such an operator, analytic hypoellipticity was already obtained in F. Treves [13] under the additional hypothesis that

$$
P(x, D) \text { is hypoelliptic with loss of one derivative. }
$$

Recently, several other studies have been made for the analytic hypoellipticity of similar operators. For example, in [7], G. Métivier extended the result of [13] to the operators with multiple characteristics assuming suitable hypoellipticity in case that the characteristic manifold is symplectic. Whereas, in [6], A. Grigis and L. P. Rothschild gave a necessary and sufficient condition for a class of operators with polynomial coefficients to be analytic hypoelliptic (see also [10] and [11] for a different approach to similar problems).

On the other hand, in [3], L. Boutet de Monvel and F. Treves obtained a necessary and sufficient condition for $P(x, D)$ to be hypoelliptic with loss of one derivative by means of the method of concatenations (introduced first by $\mathrm{F}$. Treves in [12]). According to their work, we can associate with $P(x, D)$ a sequence of operators $P^{(\nu)}, \nu \geqslant 0$, with the same principal part as $P(x, D)$, which satisfy certain relations connecting them, called concatenations. In terms of concatenations, the condition (2) can be restated by saying that the "lower order part" of every $P^{(\nu)}$ (i.e., the one

Received by the editors March 25, 1983 and, in revised form, September 15, 1983.

1980 Mathematics Subject Classification. Primary 35B65.

Key words and phrases. Analytic hypoelliptic, concatenations, double characteristic.

(C1984 American Mathematical Society $0002-9947 / 84 \$ 1.00+\$ .25$ per page 
obtained by neglecting its principal part) is elliptic of order $m-1$ on $\Sigma$. Then the main result of this work is the following:

$P(x, D)$ is analytic hypoelliptic in $\Omega$ if the "lower order part" of every $P^{(\nu)}$ is elliptic of arbitrary order less than or equal to $m-1$ at any point of $\Sigma$.

In fact, the latter is equivalent to saying that the "lower order part" of every $P^{(\nu)}$ is elliptic of order $m-1$ at any point of $\Sigma$ except possibly one of them. We may even assume that the exceptional one is not necessarily elliptic but has a microlocal analytic parametrix in some conic open neighborhood of any point in $\Sigma$.

As an immediate consequence of our result, we derive that $\square_{h}+C(z, t)$ is analytic hypoelliptic when $\square_{b}$ is the Kohn-Laplacian on the Heisenberg group $H_{n}$ and $C(z, t)$ is a real-analytic function on $H_{n}$ which does not vanish anywhere. This extends the result of E. M. Stein [9] that $\square_{h}+c$ is analytic hypoelliptic for any nonzero complex number $c$. Although the above example is only for operators on the Heisenberg group, the main theorem (cf. Theorem 3.1) can also be applied off the group as well.

Notations. We use the standard notations in pseudodifferential operator theory. Functions and distributions in this paper have their values in a large array of finite-dimensional vector spaces over $\mathbf{C}$, which we do not specify but will be clear from the context. Homogeneity always refers to the fibre variable in $T^{*} \Omega$ and "analytic" always means "real-analytic".

1. Complex coordinates and invariants attached to the operator. Throughout the paper, $\Omega$ will be an analytic manifold of dimension $N$. We shall denote by $T^{*} \Omega$ the cotangent bundle over $\Omega$, by $\dot{T}^{*} \Omega$ the complement of the zero section in $T^{*} \Omega$, and by $\omega$ the fundamental symplectic two-form on $T^{*} \Omega$. In local charts, the coordinates in $T^{*} \Omega$ always be denoted by $(x, \xi)=\left(x^{1}, \ldots, x^{N}, \xi_{1}, \ldots, \xi_{N}\right)$. For any two smooth complex-valued functions $f$ and $g$ on $T^{*} \Omega, H_{f}$ and $\{f, g\}=H_{f}(g)$ stand for the Hamilton field of $f$ and the Poisson bracket of $f$ and $g$, respectively.

We shall be concerned with analytic pseudodifferential operators with double characteristics of the type

$$
P(x, D)=I_{d} p(x, D)+Q(x, D),
$$

where the function $p(x, \xi)$ is a scalar analytic symbol, homogeneous of degree $m$ (real) in $\xi, Q(x, D)$ a classical analytic pseudodifferential operator with values in the space of $d \times d$ complex matrices whose order is at most $m-1$, and $I_{d}$ is the identity $d \times d$ matrix. For the theory of analytic symbols and corresponding operators, we refer to [1 and 14]. Furthermore, as in [13], we shall make the following hypotheses:

$$
p(x, \xi) \text { is always nonnegative on } T^{*} \Omega \text {; }
$$

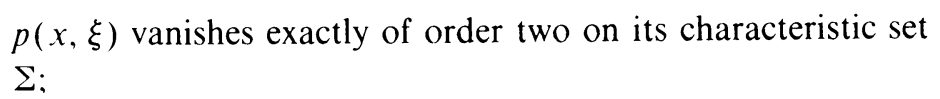
$p(x, \xi)$ vanishes exactly of order two on its characteristic set $\Sigma$;

$\Sigma$ is an analytic submanifold of $\dot{T}^{*} \Omega$ and moreover is symplectic, that is, the restriction of $\omega$ to its tangent space is nondegenerate everywhere. 
Condition (1.4) requires that the dimension of $\Sigma$ be even and hence so be its codimension: we shall set

$$
n=\frac{1}{2} \operatorname{codim} \Sigma \text {. }
$$

Let $\rho=\left(x^{0}, \xi_{0}\right)$ be an arbitrary point of $\Sigma$. Since $\omega$ is nondegenerate on $T_{\rho}(\Sigma)$, the tangent space of $\Sigma$ at $\rho$, we have

$$
T_{\rho}\left(T^{*} \Omega\right)=T_{\rho}(\Sigma) \oplus N_{\rho}(\Sigma)
$$

where $N_{\rho}(\Sigma)$ denotes the orthogonal complement of $T_{\rho}(\Sigma)$ in $T_{\rho}\left(T^{*} \Omega\right)$ with respect to $\omega$. By the assumption (1.3), we can intrinsically define a nondegenerate symmetric bilinear form on $N_{\rho}(\Sigma)$. The following definition is due to J. Sjöstrand [8] (see also [5]).

Definition 1.1. For each $\rho$ in $\Sigma$, we define a bilinear form $q_{p}(\rho)$ on $T_{\rho}\left(T^{*} \Omega\right)$ by

$$
q_{p}(\rho)\left(v_{1}, v_{2}\right)=\frac{1}{2} X_{1}\left(X_{2}(p)\right)(\rho), \quad v_{1}, v_{2} \in T_{\rho}\left(T^{*} \Omega\right),
$$

where $X_{j}$ are some vector fields defined in a neighborhood of $\rho$ with $X_{j}(\rho)=v_{j}$, $j=1,2$.

We shall denote by $Q_{p}(\rho)$ the quadratic form induced by $q_{p}(\rho)$. Moreover, since $\omega$ is nondegenerate, we have an endomorphism $A_{p}(\rho)$ of $T_{\rho}\left(T^{*} \Omega\right)$, called the Hamilton map of $q_{p}(\rho)$, defined by

$$
q_{p}(\rho)\left(v_{1}, v_{2}\right)=\omega\left(v_{1}, A_{p}(\rho)\left(v_{2}\right)\right), \quad v_{1}, v_{2} \in T_{\rho}\left(T^{*} \Omega\right) .
$$

For convenience, from now on, we shall use $q_{p}$ and $A_{p}$ instead of $q_{p}(\rho)$ and $A_{p}(\rho)$, respectively. By (1.2) and (1.3), $q_{p}$ is a well-defined symmetric bilinear form on $T_{\rho}\left(T^{*} \Omega\right)$ and $Q_{p}$ is positive-definite when it is restricted to $N_{\rho}(\Sigma)$. In fact, $Q_{p}$ is nothing but the quadratic form which begins the Taylor expansion of $p(x, \xi)$ at a point of $\Sigma$.

Now, let $\Gamma$ be an open conic neighborhood of $\rho$ in which there are $2 n$ analytic real-valued functions $u_{1}, \ldots, u_{2 n}$, with linearly independent differentials, such that $\Sigma \cap \Gamma$ is exactly defined by the equations

$$
u_{j}(x, \xi)=0, \quad j=1, \ldots, 2 n .
$$

We take $u_{j}$ to be homogeneous of degree $\frac{1}{2}$ in $\xi$. Then, in $\Gamma$, we can write

$$
p(x, \xi)=\sum q_{j k} u_{j} u_{k},
$$

where $q_{j k}$ are analytic in $\Gamma$ and homogeneous of degree $m-1$ in $\xi$. We may and shall assume that $q_{j k}=q_{k j}$ for every $j, k=1, \ldots, 2 n$. The following proposition is an analytic version of a particular case of results in [ 2 and 3 ].

Proposition 1.1. There exist complex linear combinations $z_{1}, \ldots, z_{n}$ of $u_{j}, j=$ $1, \ldots, 2 n$, with complex-valued analytic homogeneous coefficients such that

$$
p(x, \xi)=\sum a_{j k} z_{j} \bar{z}_{k}
$$


where the matrix $\left(a_{j k}\right)$ is positive-definite hermitian at each point of $\Sigma \cap \Gamma$ and its eigenvalues $\lambda_{j}, j=1, \ldots, n$, which are all strictly positive, are the invariants attached to the operator $P(x, D)$;

$$
\left\{z_{j}, z_{k}\right\}=\frac{1}{\sqrt{-1}}\left\{z_{j}, \bar{z}_{k}\right\}-\delta_{j k}=0 \text { in } \Gamma
$$

( possibly after shrinking $\Gamma$ ), $j, k=1, \ldots, n$.

To prove the above proposition, we need some results from linear algebra. Let $E$ be a real vector space of dimension $2 n, \omega$ a real symplectic form on $E$, and $Q$ a positive-definite quadratic form on $E$. We shall denote by $q$ the polarization of $Q$. Extend both $q$ and $\omega$ to $E_{\mathrm{C}}=E \otimes \mathbf{C}$, the complexification of $E$, as bilinear forms. Then we have an endomorphism $A$ of $E_{\mathbf{C}}$, the Hamilton map of $q$, defined by

$$
q(u, v)=\omega(u, A v), \quad u, v \in E_{\mathbf{C}} .
$$

For the proof of the following lemma, we refer to [2] (see also $[3,5])$.

LEMMA 1.1. With the above notations, we have that

(1) there are $n$ positive real numbers $\lambda_{j}, j=1, \ldots, n$, not necessarily distinct, such that the eigenvalues of $A$ are precisely $\pm \sqrt{-1} \lambda_{j}, j=1, \ldots, n$;

(2) there exist complex coordinates $z_{1}, \ldots, z_{n}$ in $E_{\mathbf{C}}$, unique up to linear combinations, such that

$$
\begin{aligned}
& \omega\left(z_{j}, z_{k}\right)=\frac{1}{\sqrt{-1}} \omega\left(z_{j}, \bar{z}_{k}\right)-\delta_{j k}=0, \quad j, k=1, \ldots, n ; \\
& Q=\sum a_{j k} z_{j} \bar{z}_{k},\left(a_{j k}\right) \text { a positive-definite hermitian matrix with } \\
& \lambda_{j} \text { in }(1) \text { as its eigenvalues. }
\end{aligned}
$$

Proof of Proposition 1.1. If $z_{k}=\sum \alpha_{k j} u_{j}$, then $d z_{k}=\sum \alpha_{k j} d u_{j}$ and $\left\{z_{k}, z_{l}\right\}=$ $\sum \alpha_{k i} \alpha_{l j}\left\{u_{i}, u_{j}\right\}$ on $\Sigma \cap \Gamma$ because $u_{j}$ vanish on $\Sigma \cap \Gamma$. Applying Lemma 1.1 with $E=\mathbf{R}^{2 n}, Q$ the quadratic form with the matrix $\left(q_{j k}\right)$, and $\omega$ the symplectic form whose matrix is the inverse of $\left(\left\{u_{j}, u_{k}\right\}\right)$, we get $z_{j}, j=1, \ldots, n$, which satisfy (1.11) and (1.12) only in $\Sigma \cap \Gamma$. But if we denote by $x_{j}$ and $y_{j}$ the real and imaginary parts of $z_{j},(1.12)$ means

$$
\left\{x_{j}, x_{k}\right\}=\left\{y_{j}, y_{k}\right\}=\left\{x_{j}, y_{k}\right\}+\frac{1}{2} \delta_{j k}=0 \text { in } \Sigma \cap \Gamma, j, k=1, \ldots, n .
$$

Hence, by the classical theorem of Darboux for the real symplectic coordinates, we can choose $z_{j}$ so that they satisfy (1.12) in $\Gamma$ if it is sufficiently narrow about $\Sigma \cap \Gamma$.

REMARK 1.1. The vector space spanned by $d z_{j}$ at each point of $\Sigma \cap \Gamma$ is uniquely determined by (1.11) and (1.12).

Proposition 1.2. In a possibly smaller conic open set $\Gamma$, we can construct $n$ analytic pseudodifferential operators $Z_{j}$ with principal symbols $z_{j}$ in $\Gamma$ which satisfy the following commutation relations:

$\left[Z_{j}, Z_{k}\right]$ and $\left[Z_{j}, Z_{k}^{*}\right]-I \delta_{j k}$ are analytic regularizing in $\Gamma$, $j, k=1, \ldots, n$, where $I$ is the identity operator. 
As usual, the proof is by successive approximations and can be found in [3]. In [2], L. Boutet de Monvel has obtained a more precise result than this using Fourier integral operators in the $C^{\infty}$-category. For the same result in the analytic category, see [13, Chapter 1].

Let $Z_{j}$ be the operators in Proposition 1.2 and let $A_{j k}$ be the analytic pseudodifferential operators in $\Gamma$ with principal symbols $a_{j k}$. Let us set

$$
Z_{0}=P(x, D)-I_{d}\left(\sum A_{j k} Z_{k}^{*} Z_{j}\right) \text { in } \Gamma .
$$

Then $Z_{0}$ is a $d \times d$ matrix with analytic pseudodifferential operators in $\Gamma$ as entries whose order is at most $m-1$. We shall denote by $\sigma_{0}=\sigma_{0}(x, \xi)$ the restriction to $\Sigma \cap \Gamma$ of the principal symbol of $Z_{0}$ regarded as an operator of order $m-1$ in $\Gamma$. If we set

$$
W_{j}=\sum A_{j k} Z_{k}^{*}, \quad j=1, \ldots, n,
$$

then we have, in $\Gamma$,

$$
P(x, D)=I_{d}\left(\sum W_{j} Z_{j}\right)+Z_{0} .
$$

Let $p_{m}+p_{m-1}+\cdots$ be the complete symbol of $P$ in a local coordinate system $(x, \xi)$, where $p_{j}(x, \xi)$ is homogeneous of degree $j$ in $\xi$. Then

$$
\begin{aligned}
p_{m-1} & =\sigma_{0}(x, \xi)+I_{d}\left(\frac{1}{\sqrt{-1}} \sum \frac{\partial w_{j}}{\partial \xi_{k}} \frac{\partial z_{j}}{\partial x^{k}}\right) \\
& =\sigma_{0}(x, \xi)+\frac{1}{2 \sqrt{-1}} \sum \frac{\partial^{2} p_{m}(x, \xi)}{\partial \xi_{k} \partial x^{k}}+\frac{1}{2 \sqrt{-1}} I_{d}\left(\sum\left\{w_{j}, z_{j}\right\}\right) \\
& =\sigma_{0}(x, \xi)+\frac{1}{2 \sqrt{-1}} \sum \frac{\partial^{2} p_{m}(x, \xi)}{\partial \xi_{k} \partial x^{k}}-I_{d}\left(\frac{1}{2} \sum \lambda_{j}\right) \text { on } \Sigma \cap \Gamma,
\end{aligned}
$$

where $w_{j}$ are the principal symbol of $W_{j}$ and $\lambda_{j}$ are the eigenvalues of the matrix $\left(a_{j k}\right)$ (cf. Proposition 1.1). Therefore we have another invariant of $P(x, D)$ :

$$
\sigma_{0}(x, \xi)=\sigma_{\text {sub }}(P)+I_{d}\left(\frac{1}{2} \sum \lambda_{j}\right),
$$

where

$$
\sigma_{\text {sub }}(P)=p_{m-1}(x, \xi)-\frac{1}{2 \sqrt{-1}} \sum \frac{\partial^{2} p_{m}(x, \xi)}{\partial \xi_{k} \partial x^{k}}
$$

is the subprincipal symbol of $P(x, D)$. In fact, this invariant is a function defined on $\Sigma$, whose value lies in the space of $d \times d$ complex matrices.

Remark 1.2 . Note that the adjoint $P^{*}$ of $P$ is an operator of exactly the same type as $P$ and the invariants associated with $P^{*}$ are the complex conjugates of those associated with $P$.

2. Concatenations and hypoellipticity with loss of one derivative. Throughout this section, we restrict our attention to the conic open set $\Gamma$ in which we have (1.19). We are going to construct a sequence of operators, which satisfy certain relations, called concatenations, through which (analytic) hypoellipticity can be transmitted backward 
and then give the connection between the concatenations and the hypoellipticity with loss of one derivative. The latter means, in our case, that for any open subset $U$ of $\Omega$, any real number $s$, and any distribution $u$ in $U$,

$$
P(x, D)(u) \in H_{\mathrm{loc}}^{s}\left(U ; \mathbf{C}^{d}\right) \text { implies } u \in H_{\mathrm{loc}}^{s+m-1}\left(U ; \mathbf{C}^{d}\right) .
$$

We mainly follow the arguments in [3] (see also [12 and 13] for the method of concatenations). We shall now make the additional hypothesis that

$$
Z_{0} \text { is elliptic (of arbitrary order } \leqslant m-1 \text { ) in } \Gamma \text {. }
$$

Let $Z_{0}^{-1}$ be the inverse of $Z_{0}$ in $\Gamma$ (in the algebra of analytic pseudodifferential operators) and let us form

$$
Z_{j}^{\prime}=Z_{0} Z_{j} Z_{0}^{-1}, \quad j=1, \ldots, n .
$$

Then we have

$$
\begin{aligned}
Z_{j}^{\prime} P & =\left(\sum Z_{0} Z_{j} Z_{0}^{-1} W_{k} Z_{k}\right)+Z_{0} Z_{j} \\
& =I_{d}\left(\sum Z_{j} W_{k} Z_{k}\right)+Z_{0} Z_{j}+\sum\left(Z_{0}\left[Z_{j}, Z_{0}^{-1}\right] W_{k}\right) Z_{k} \\
& =\left\{I_{d}\left(\sum W_{k} Z_{k}\right)+Z_{0}\right\} Z_{j}+\sum\left(I_{d}\left[Z_{j}, W_{k}\right]+Z_{0}\left[Z_{j}, Z_{0}^{-1}\right] W_{k}\right) Z_{k},
\end{aligned}
$$

where "=" must be understood in the sense of being equal modulo analytic regularizing operators in $\Gamma$. If we set $P_{j k}=\delta_{j k} P+I_{d}\left[Z_{j}, W_{k}\right]+Z_{0}\left[Z_{j}, Z_{0}^{-1}\right] W_{k}$, then we have

$$
Z_{j}^{\prime} P=\sum P_{j k} Z_{k}, \quad j=1, \ldots, n .
$$

Let $P^{(1)}$ denote the $n d \times n d$ system $\left(P_{j k}\right)$ and $Z$ (resp. $\left.Z^{(1)}\right)$ the column with $n$ entries, which are $d \times d$ systems $I_{d} Z_{j}$ (resp. $I_{d} Z_{j}^{\prime}$ ). Then we can rewrite (2.4) in the form

$$
Z^{(1)} P=P^{(1)} Z
$$

On the other hand, we can write

$$
P^{(1)}=I_{n d}\left(\sum W_{k} Z_{k}\right)+Z_{0}^{(1)},
$$

where $Z_{0}^{(1)}$ is of order at most $m-1$ in $\Gamma$.

Now, assuming that $Z_{0}^{(1)}$ is also elliptic in $\Gamma$, we can repeat the same argument as above inductively and get a sequence of operators $P^{(\nu)}, \nu \geqslant 0$, with $P^{(0)}=P$, which form concatenations

$$
Z^{(\nu+1)} P^{(\nu)}=P^{(\nu+1)} Z
$$

where

$Z$ is the column with $n$ entries, respectively equal to $I_{n} v_{d} Z_{j}$, $j=1, \ldots, n$,

$$
\begin{gathered}
Z_{j}^{(\nu+1)}=Z_{0}^{(\nu)} Z_{j}\left(Z_{0}^{(\nu)}\right)^{-1}, \quad j=1, \ldots, n, \\
P^{(\nu)}=I_{n} \nu_{d}\left(\sum W_{k} Z_{k}\right)+Z_{0}^{(\nu)} \quad\left(Z_{0}^{(0)}=Z_{0}\right) .
\end{gathered}
$$

Of course, in the above arguments, we have assumed that

$$
Z_{0}^{(\mu)} \text { is elliptic (of arbitrary order } \leqslant m-1 \text { ) in } \Gamma, \mu=0, \ldots, \nu \text {. }
$$


Our argument might require successive shrinkings of $\Gamma$, which does not matter to us since we shall use only a finite number of steps in the concatenations. We are now ready to give the connection between the concatenations and the hypoellipticity with loss of one derivative for $P(x, D)$. Let us consider a special case of (2.11):

$$
Z_{0}^{(\mu)} \text { is elliptic of order } m-1 \text { in } \Gamma \text {. }
$$

In this case, (2.12) is equivalent (possibily after shrinking $\Gamma$ ) to

$$
Z_{0}^{(\mu)} \text { is elliptic of order } m-1 \text { in } \Sigma \cap \Gamma \text {. }
$$

Let us denote by $\sigma_{0}^{(\mu)}$ the restriction to $\Sigma \cap \Gamma$ of the principal symbol of $Z_{0}^{(\mu)}$ as an operator of order $m-1$ and set

$$
\theta_{j k}=\sigma\left(\left[Z_{j}, W_{k}\right]\right) \mid \Sigma \cap \Gamma, \quad j, k=1, \ldots, n .
$$

For $\mu=1$, from the definition of $P_{j k}$, we have

$$
\left(\sigma_{0}^{(1)}\right)_{j k}=\delta_{j k} \sigma_{0}^{(0)}+\theta_{j k} I_{d}, \quad j, k=1, \ldots, n .
$$

That is,

$$
\sigma_{0}^{(1)}=\sigma_{0}^{(0)} \otimes I_{n}+I_{d} \otimes \Theta,
$$

where $\Theta$ is the matrix $\left(\theta_{j k}\right)$. More generally, for any $\mu \geqslant 1$,

$$
\sigma_{0}^{(\mu)}=\sigma_{0}^{(\mu-1)} \otimes I_{n}+I_{n^{\mu-1} d} \otimes \Theta .
$$

The hypothesis (2.13) simply means that the eigenvalues of $\sigma_{0}^{(\mu)}$, which depends on the points of $\Sigma \cap \Gamma$, are all distinct from zero. From (2.17), we can easily see that any eigenvalue of $\sigma_{0}^{(\mu)}$ is a sum of an eigenvalue of $\sigma_{0}^{(\mu-1)}$ and an eigenvalue of $\Theta$. By induction on $\mu$, we have

$$
\operatorname{Spec} \sigma_{0}^{(\mu)}=\operatorname{Spec} \sigma_{0}^{(0)}+\underbrace{\operatorname{Spec} \Theta+\cdots+\operatorname{Spec} \Theta,}_{\mu \text {-times }}
$$

where the plus signs on the right stand for the addition of complex numbers. On the other hand, from (1.18) and (2.14), we see that the matrix $\Theta$ is just the transpose of $\left(a_{j k}\right)$ over $\Sigma \cap \Gamma$ and hence the eigenvalues of $\Theta$ are the positive real numbers $\lambda_{j}$, $j=1, \ldots, n$, introduced in Proposition 1.1.

Therefore, when $\mu$ ranges over $Z_{+}$and $\Gamma$ ranges over a covering of $\Sigma$ by open cones, the hypothesis (2.13) is equivalent to the following property:

For no point $(x, \xi)$ in $\Sigma$ and for no $n$-tuple of nonnegative integers $r=\left(r_{1}, \ldots, r_{n}\right)$, does the $d \times d$ matrix $\sigma_{0}(x, \xi)$ (let us remind that $\sigma_{0}(x, \xi)$ is an invariant of $P(x, D)$ defined on $\left.\Sigma\right)$ have the eigenvalues $-\Sigma r_{j} \lambda_{j}$.

As is now well known (cf. $[2,3,5,8])$, condition (2.19) is necessary and sufficient for $P(x, D)$ to be hypoelliptic with loss of one derivative.

REMARK 2.1. The above construction of the concatenations and the observations for the condition (2.13) have already been done in [3] for a larger class of operators in the $C^{\infty}$-category. However, as long as we are concerned with analytic hypoellipticity, we can also construct the same concatenations (2.7) under a much weaker condition that every $Z_{0}^{(\mu)}$ has an analytic parametrix in some open conic neighborhood of each point of $\Sigma$. 
REMARK 2.2. According to Remark 1.2, $P$ is hypoellitpic with loss of one derivative if and only if so is the adjoint $P^{*}$ of $P$.

3. Analytic hypoellipticity. Throughout this section, we are going to assume that $P(x, D)$ is given by (1.19) not only in $\Gamma$ but in the whole of $T^{*} \Omega$. This is possible since analytic hypoellipticity is purely a local matter and outside $\Sigma, P(x, D)$ is elliptic. Moreover, after a preliminary shrinking of $\Gamma$ if necessary, we may and shall assume that $\Sigma$ is conically compact. Now, let us consider the following condition for $P(x, D)$ under which we can construct concatenations $(2.7)$ :

Every $Z_{0}^{(\nu)}, \nu \geqslant 0$, has a microlocal analytic parametrix in some conic open neighborhood of each point in $\Sigma$.

It follows from the formula (2.18) and the conical compactness of $\Sigma$ that all but a finite number of $Z_{0}^{(\nu)}$ are elliptic of order $m-1$ on $\Sigma$ when $P(x, D)$ satisfies condition (3.1). Now, we can state and prove the main result of this work.

THEOREM 3.1. If $P(x, D)$ satisfies condition $(3.1)$, then $P(x, D)$ is analytic hypoelliptic in $\Omega$.

First of all, let us recall the main result of [13].

Let $P(x, D)$ be given by (1.1) which satisfies (1.2), (1.3), and (1.4). If it is hypoelliptic with loss of one derivative, then any point of $\Sigma$ has an open conic neighborhood, say $\Gamma^{0}$, in which $P(x, D)$ is analytic hypoelliptic, that is, for any $u \in \varepsilon^{\prime}\left(\Omega: \mathbf{C}^{d}\right)$, $W F_{a}(u) \cap \Gamma^{0}=W F_{a}(P u) \cap \Gamma^{0}$ (cf. [13, Theorem 1.4.2]), where $W F_{a}(u)$ stands for the analytic wave-front set of $u$.

(The proof is by construction of a microlocal analytic parametrix of $P(x, D)$ near every point of $\Sigma$.)

Then, recalling that the base projection of analytic wave-front set of a distribution is its analytic singular-support, the analytic hypoellipticity of $P(x, D)$ is just a local version of the above result.

The following lemma will show that the analytic hypoellipticity can be transmitted backward along the concatenations.

LEMma 3.1. Suppose $P(x, D)$ satisfies condition (3.1). If, for some $\mu \geqslant 0, P^{(\mu+1)}$ is analytic hypoelliptic in $\Omega$, then so is $P^{(\mu)}$.

Proof. Let $u$ be a distribution with compact support in $\Omega$ for which $P^{(\mu)}(u)$ is analytic in an open set $U$ in $\Omega$. Since the characteristic set of $P^{(\mu)}$ is exactly $\Sigma$, $W F_{a}(u) \cap T^{*} U$ is contained in $\Sigma$. On the other hand, from $(2.7), P^{(\mu+1)} Z(u)=$ $Z^{(\mu+1)} P^{(\mu)}(u)$ and so $Z(u)$ is analytic in $U$ because $P^{(\mu+1)}$ is analytic hypoelliptic in $\Omega$. That means, from the definition of $Z$ (cf. (2.8)), all the $Z_{j}(u)$ are analytic in $U$. Therefore,

$$
Z_{0}^{(\mu)}(u)=P^{(\mu)}(u)-I_{n} \mu_{d}\left(\sum W_{k} Z_{k}\right)(u)
$$

(cf. (2.10)) is also analytic in $U$. But since $Z_{0}^{(\mu)}$ is analytic hypoelliptic near every point of $\Sigma$ by the hypothesis (3.1), we have $W F_{a}(u) \cap T^{*} U \cap \Sigma=\varnothing$, which implies 
that $W F_{a}(u) \cap T^{*} U=\varnothing$ (recalling that $W F_{a}(u) \cap T^{*} U$ is contained in $\Sigma$ ). That is, $u$ is also analytic in $U$.

REMARK 3.1. Repeated applications of Lemma 3.1 show that if $P(x, D)$ satisfies condition (3.1) and if $P^{(\mu+1)}$ is analytic hypoelliptic in $\Omega$ for some $\mu \geqslant 0$, then so are $P=P^{(0)}, \ldots, P^{(\mu)}$.

Proof of THEOREM 3.1. As we have mentioned in the beginning of this section, under the condition (3.1), there is a nonnegative integer $\mu_{0}$ such that all the $Z_{0}^{(\mu)}$, $\mu>\mu_{0}$, are elliptic of order $m-1$ 'on $\Sigma$. Then, in particular, $P^{\left(\mu_{0}+1\right)}$ satisfies condition (2.13) and hence is hypoelliptic with loss of one derivative since it has the same scalar principal part as that of $P(x, D)$. Therefore, thanks to $(3.2), P^{\left(\mu_{0}+1\right)}$ is analytic hypoelliptic in $\Omega$. Due to Remark 3.1 , this proves that $P(x, D)$ itself is analytic hypoelliptic in $\Omega$.

Noting that condition (2.11) is a special case of (3.1), we get immediately the following corollary.

Corollary 3.1. If $P(x, D)$ satisfies condition $(2.11)$, then $P(x, D)$ is analytic hypoelliptic in $\Omega$. Moreover the condition (2.11) is satisfied if $Z_{0}$ is elliptic of order strictly less than $m-1$ in some conic open neighborhood of each point in $\Sigma$.

Proof. We only need to prove the second statement of the above corollary. Let us consider $\sigma_{0}^{(\mu)}, \mu \geqslant 0$, which is the restriction to $\Sigma$ of the principal symbol of $Z_{0}^{(\mu)}$ as an operator of order $m-1$. Then $\sigma_{0}^{(0)}$ is identically zero on $\Sigma$ by the assumption. Therefore, from (2.18), any eigenvalue of $\sigma_{0}^{(\mu)}, \mu>0$, is of the form $\sum r_{j} \lambda_{j}$, where $r=\left(r_{1}, \ldots, r_{n}\right) \in Z_{+}^{n},|r|=r_{1}+\cdots+r_{n}=\mu$, and $\lambda_{j}$ are the eigenvalues of $\left(a_{j k}\right)$ (cf. (1.11)). Since all the $\lambda_{j}$ are strictly positive, 0 cannot be an eigenvalue of $\sigma_{0}^{(\mu)}$ for $\mu>0$. That is, all the $Z_{0}^{(\mu)}, \mu>0$, are elliptic of order $m-1$ on $\Sigma$. Hence condition (2.11) is satisfied (cf. (2.13)).

REMARK 3.2. Although our main concern is analytic hypoellipticity, we incidently get $C^{\infty}$-hypoellipticity of $P(x, D)$, without any significant change of previous arguments, under the same hypotheses as in Theorem 3.1 or Corollary 3.1 except the fact that $P(x, D)$ can be a usual $\left(C^{\infty}\right)$ pseudodifferential operator instead of an analytic one.

4. Application. In this section, as an application of our results, we will consider an operator defined on the Heisenberg group $H_{n}$ which arises as the boundary of the generalized upper half-space in $\mathbf{C}^{n+1}$,

$$
D=\left\{\left(z_{1}, \ldots, z_{n+1}\right) \in \mathbf{C}^{n+1} ; \operatorname{Im} z_{n+1}>\left|z_{1}\right|^{2}+\cdots+\left|z_{n}\right|^{2}\right\} .
$$

Its boundary can be identified with $\mathbf{C}^{n} \times \mathbf{R}^{1}$, which has a structure of Heisenberg group $H_{n}$ on which we have the following left-invariant complex vector fields (denoting by $(z, t)=\left(z_{1}, \ldots, z_{n}, t\right)$ the coordinates in $\left.H_{n}\right)$ :

$$
\begin{aligned}
& Z_{j}=\frac{\partial}{\partial z_{j}}+\sqrt{-1} \bar{z}_{j} \frac{\partial}{\partial t}, \\
& \bar{Z}_{j}=\frac{\partial}{\partial \bar{z}_{j}}-\sqrt{-1} z_{j} \frac{\partial}{\partial t}, \quad j=1, \ldots, n,
\end{aligned}
$$


which satisfies the following commutation relations:

$$
\left[Z_{j}, Z_{k}\right]=0, \quad\left[Z_{j}, Z_{k}^{*}\right]=-2 D_{t} \delta_{j k}, \quad j, k=1, \ldots, n .
$$

Under the identification of $H_{n}$ with $\partial D, \bar{Z}_{j}$ annihilate holomorphic functions restricted to $\partial D$ and hence induce a complex $\bar{\partial}_{b}$, called the tangential CauchyRiemann complex on $H_{n}$. It is defined on the functions on $H_{n}$ by the formula

$$
\bar{\partial}_{b} f=\sum\left(\bar{Z}_{j} f\right) d \bar{z}_{j},
$$

which extends as a derivation mapping $(0, q)$-forms to $(0, q+1)$-forms on $H_{n}$. Then we have the (Kohn-) Laplacian $\square_{b}$ associated to the $\bar{\partial}_{b}$-complex

$$
\square_{b}=\bar{\partial}_{b} \bar{\partial}_{b}^{*}+\bar{\partial}_{b}^{*} \bar{\partial}_{b},
$$

where $\bar{\partial}_{b}^{*}$ is the formal adjoint of $\bar{\partial}_{b}$ with respect to $L^{2}$-inner product. As is well known, the restriction of $\square_{b}$ to the functions on $H_{n}$, which is given by

$$
\square_{b}=-\sum Z_{j} \bar{Z}_{k},
$$

is neither locally solvable nor hypoelliptic (see, for example, [4]) and its characteristic manifold $\Sigma$ is symplectic, which is a reflection of the fact that $H_{n}$ is strongly pseudoconvex.

In [9], E. M. Stein restricted his attention to the left-invariant operators on $H_{n}$ and showed that $\square_{b}+c$ is $C^{\infty}$ - and analytic hypoelliptic for any nonzero complex number $c$ when it acts on the functions on $H_{n}$. We see that one gets the following as an immediate consequence of Corollary 3.1 and Remark 3.2.

THEOREM 4.1. Let $C(z, t)$ be any real-analytic (resp. $C^{\infty}$-) complex-valued function defined on $H_{n}$. If it is nowhere vanishing on $H_{n}$, then the operator $\square_{b}+C(z, t)$ is analytic hypoelliptic (resp. $C^{\infty}$-hypoelliptic) on $H_{n}$.

Proof. Let us denote by $P$ the operator $\square_{b}+C(z, t)$. In order to apply Corollary 3.1 , we need the commutation relations (1.16) rather than (4.2). Since

$$
D_{t}=\frac{1}{\sqrt{-1}} \frac{\partial}{\partial t}
$$

is elliptic on $\Sigma$, in a conic open neighborhood of any point in $\Sigma$, we can replace $Z_{j}$ (cf. (4.1)) by $\left(2\left|D_{t}\right|\right)^{-1 / 2} Z_{j}$ so that they satisfy (1.16) (up to sign). It suffices to prove the theorem for $\left(2\left|D_{t}\right|\right)^{-1} P$, which follows immediately from Corollary 3.1 .

REMARK 4.1. We can also consider analytic hypoellipticity of $\square_{b}$ acting on $(0, q)$-forms on $H_{n}$ for $q>0$. As is well known, $\square_{b}=I L_{\alpha}$, where $\alpha=n-2 q$ when it acts on $(0, q)$-forms,

$$
L_{\alpha}=-\frac{1}{2} \sum\left(Z_{j} \bar{Z}_{j}+\bar{Z}_{j} Z_{j}\right)+\sqrt{-1} \alpha T \quad\left(T=\frac{\partial}{\partial t}\right),
$$

and $I$ is the identity matrix of an appropriate degree which depends on $q$. Moreover, according to (2.19), $L_{\alpha}$ is hypoelliptic with loss of one derivative if and only if $n \pm \alpha$ is not an even integer $\leqslant 0$. Hence $\square_{b}$ is analytic hypoelliptic when it acts on $(0, q)$-forms for $q \neq 0, n$. This is already pointed out by F. Treves in [13] (see also [10 and 11] for a different proof (using $L^{2}$-methods) of analytic hypoellipticity of $\left.\square_{b}\right)$. 
ACKNOWLEDGEMENTs. The author would like to express his deep graditude to Professor François Treves for suggesting the problem and for his valuable advice throughout the whole work.

\section{REFERENCES}

1. L. Boutet de Monvel, Opérateurs pseudodifferentiels analytiques et opérateurs d'ordre infini, Ann. Inst. Fourier (Grenoble) 22 (1972), 229-268.

2. , Hypoelliptic operators with double characteristics and related pseudodifferential operators, Comm. Pure Appl. Math., 27 (1974), 585-639.

3. L. Boutet de Monvel and F. Treves, On a class of systems of pseudodifferential equations with double characteristics, Comm. Pure Appl. Math. 27 (1974), 59-89.

4. P. C. Greiner, and E. M. Stein, Estimates for the $\bar{\partial}$-Neumann problem, Math. Notes No. 19, Princeton Univ. Press, Princeton, N.J., 1977.

5. A. Grigis, Hypoellipticité et paramétrix pour des opérateurs pseudodifférentiels à caractéristiques doubles, Asterisque 34-35 (1976), 183-205.

6. A. Grigis and L. P. Rothschild, A criterion for analytic hypoellipticity of a class of differential operators with polynomial coefficients, preprint.

7. G. Métivier, Analytic hypoellipticity for operators with multiple characteristics, Comm. Partial Differential Equations 6 (1981), 1-90.

8. J. Sjöstrand, Parametrices for pseudodifferential operators with multiple characteristics, Ark. Mat. 12 (1974), 85-130.

9. E. M. Stein, An example on the Heisenberg group related to the Lewy operator, preprint.

10. D. S. Tartakoff, Local analytic hypoellipticity for $\square_{b}$ on non-degenerate Cauchy-Riemann manifolds, Proc. Nat. Acad. Sci. U.S.A. 75 (1978), 3027-3028.

11. __ Elementary proofs of analytic hypoellipticity for $\square_{h}$ and the $\bar{\partial}$-Neumann problem, Asterisque 89-90 (1981), 85-116.

12. F. Treves, Concatenations of second order evolution equations applied to local solvability and hypoellipticity, Comm. Pure Appl. Math. 24 (1973), 201-250.

13. Analytic hypoellipticity of a class of pseudodifferential operators with double characteristics and applications to the $\bar{\partial}$-Neumann problem, Comm. Partial Differential Equations 3 (1978), 475-642.

14. , Introduction to pseudodifferential and Fourier integral operators, Vols. 1 \& 2, Plenum Press, New York, 1980.

Department of Mathematics, Rutgers University, New Brunswick, New Jersey 08903

Current address: Department of Mathematics, University of Oklahoma, Norman, Oklahoma 73019 